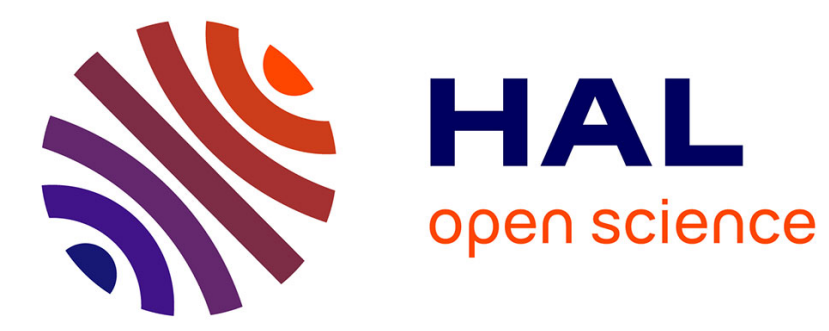

\title{
Polarization and Geometry in the n-Site DAFS Problem
} J. Cross, J. Rehr, L. Sorensen

\section{To cite this version:}

J. Cross, J. Rehr, L. Sorensen. Polarization and Geometry in the n-Site DAFS Problem. Journal de Physique IV Proceedings, 1997, 7 (C2), pp.C2-761-C2-762. 10.1051/jp4:1997229 . jpa-00255308

\section{HAL Id: jpa-00255308 https://hal.science/jpa-00255308}

Submitted on 1 Jan 1997

HAL is a multi-disciplinary open access archive for the deposit and dissemination of scientific research documents, whether they are published or not. The documents may come from teaching and research institutions in France or abroad, or from public or private research centers.
L'archive ouverte pluridisciplinaire HAL, est destinée au dépôt et à la diffusion de documents scientifiques de niveau recherche, publiés ou non, émanant des établissements d'enseignement et de recherche français ou étrangers, des laboratoires publics ou privés. 


\title{
Polarization and Geometry in the $n$-Site DAFS Problem
}

\author{
J.O. Cross, J.J. Rehr* and L.B. Sorensen* \\ Naval Research Laboratory, Washington DC 20375, U.S.A. \\ * Department of Physics, Box 351560, University of Washington, Seattle, WA 98195, U.S.A.
}

\begin{abstract}
The use of diffraction anomalous fine-structure (DAFS) as a means to isolate site-specific XAFS-like response functions in low symmetry materials requires careful consideration of the polarization dependence of the anomalous scattering amplitudes. Starting from the second order matrix elements that describe the anomalous scattering, we use the Rehr-Albers scattering matrix formalism to describe the most general DAFS polarization tensor and make several conclusions about the polarization dependence of DAFS. In this short report, we limit our discussion to practical issues of experimental geometry for the case of DAFS from a material with multiple inequivalent sites in the unit cell.
\end{abstract}

\section{INTRODUCTION}

It is well known from $x$-ray absorption studies that both the fine-structure and the position of the absorption edge can have a strong dependence on the polarization direction of the incident photons. In the analogous resonant diffraction experiments, the scattered photon polarization can be different from the incident photon polarization, either by diffracting out of the plane defined by the incident polarization, or by resonant scattering that rotates the sense of polarization. The optical theorem relates the fine structure in the elastic scattering channel to the absorption fine structure in the forward scattering direction, where incident and outgoing photon wavevectors $\mathbf{k}$ and $\mathbf{k}^{\prime}$ are equal. For measurements made at non-zero wavevector transfer $\mathbf{Q}=\mathbf{k}-\mathbf{k}^{\prime} \neq 0$, the independent $\mathbf{k}$ and $\mathbf{k}^{\prime}$ dependence of the resonant elastic scattering is negligible when the dipole approximation holds. However, an independent $\hat{\mathbf{e}}$ and $\hat{\mathbf{e}}^{\prime}$ dependence remains that is generally different for each photoelectron scattering path contributing to the fine structure. In this short report, we discuss the relationship between XAFS and DAFS polarization dependence and some implications for DAFS experiments.

\section{POLARIZATION DEPENDENCE OF XAFS AND DAFS}

We consider photoabsorption as an incident photon interacting with a single deep core electron. The total absorption cross-section is proportional to the squared magnitude of the sum over photoelectron final states in the first order matrix elements $\left\langle\psi_{f}|\mathbf{p} \cdot \mathbf{A}| \psi_{i}\right\rangle$ of the interaction Hamiltonian. Expanding Fermi's golden rule in terms of the one-particle Green's function $G^{+}\left(E_{i}+\hbar \omega\right)$, the absorption cross-section is written

$$
\sigma=-\left(\frac{4 \pi e^{2}}{\omega m^{2} c}\right) \operatorname{Im}\left[\left\langle\psi_{i}\left|\hat{\mathbf{e}}^{*} \cdot \mathbf{p}^{\prime} e^{-i \mathbf{k} \cdot \mathbf{r}} G^{+}\left(E_{i}+\hbar \omega\right) \hat{\mathbf{e}} \cdot \mathbf{p} e^{i \mathbf{k} \cdot \mathbf{r}}\right| \psi_{i}\right\rangle\right]
$$

where $\psi_{i}$ is the initial core state. Within the dipole approximation, the dependence on $\mathbf{k}$ vanishes, however, the dependence on $\hat{\mathbf{e}}$, which is generally complex, remains. In Eq.(1), the polarization vector $\hat{\mathbf{e}}$ of the initial state photon appears twice. In the multiple scattering expansion of Eq. (1), the polarization dependence the XAFS can be considered as the projection of the incident photon polarization onto the first and last legs of each $n$-leg scattering path.

The resonant elastic scattering amplitude is second order in the interaction Hamiltonian. The matrix elements which give the fine structure in DAFS may be written in terms of the same Green's function expansion as Eq. (1) by

$$
\Delta f=-\left(\frac{e}{m c}\right)^{2}\left\langle\psi_{i}\left|\hat{\mathbf{e}}^{\prime *} \cdot \mathbf{p}^{\prime} e^{-i \mathbf{k}^{\prime} \cdot \mathbf{r}^{\prime}} G^{+}\left(E_{i}+\hbar \omega\right) \hat{\mathbf{e}} \cdot \mathbf{p} e^{i \mathbf{k} \cdot \mathbf{r}}\right| \psi_{i}\right\rangle
$$

This expression describes the amplitude for resonant scattering and, although Eq. (2) is similar in appearance to Eq. (1), resonant scattering is a two photon process and $\mathbf{k}$ and $\hat{\mathbf{e}}$ are independent of $\mathbf{k}^{\prime}$ and $\hat{\mathbf{e}}^{\prime}$. The same multiple scattering picture applies to DAFS except that the path polarization dependence goes as the projection of the initial state polarization onto the first leg of the path and the final state polarization onto the last leg of the path.

Within the Rehr-Albers formalism and the dipole approximation, the XAFS polarization tensor $\chi_{\alpha \beta}$ is calculated by expanding $G^{+}$in local $|L, \mathbf{R}\rangle$ states, writing the matrix elements in terms of a radial part and an angular momentum 
part. The polarization dependence of the DAFS fine structure is expressed in the terms of the fine structure tensor [1] as

$$
\chi=\left(\hat{e}_{\alpha}^{\prime}\right)^{*} \hat{e}_{\beta} \chi \alpha \beta .
$$

The only difference between Eq. (3) and the expression for the XAFS polarization dependence is that for DAFS $\hat{\mathbf{e}}$ and $\hat{\mathbf{e}}^{\prime}$ are independent vectors which may or may not be equal, whereas for XAFS $\hat{\mathbf{e}}=\hat{\mathbf{e}}^{\prime}$. Thus the computer program FEFF, based on the Rehr-Albers formalism, is able to calculate the full DAFS polarization dependence with the use of a generalized termination matrix.

\section{PRACTICAL CONSIDERATIONS FOR DAFS EXPERIMENTS}

One of the most useful applications of DAFS is to isolate the extended fine-structure and near-edge resonance spectra from atoms in inequivalent sites of a complex unit cell. In low symmetry materials, site separation is obtained by making linear combinations of the mixed anomalous scattering amplitudes $\Delta f(\mathbf{Q}, E)$ from several reflections. When the sublattice of resonant atoms is centrosymmetrie, the complete amplitudes may be obtained by self-consistent iterative application of the Kramers-Krönig dispersion relations [2] or by a spline background removal with proper normalization, which gives a close approximation in most cases. The complex $\Delta f(\mathbf{Q}, E)$ is a linear sum of the individual $\Delta f(E)$ from each inequivalent site normalized to the single atom response

$$
\Delta f(\mathbf{Q}, E)=\sum_{j=1}^{n} W_{j}(\mathbf{Q}) \Delta f_{j}(E)
$$

for $n$ unique sites in the unit cell, and with

$$
\Delta f_{j}(E)=f_{a}^{\prime}(E)+i f_{a}^{\prime \prime}(E)+f_{r}^{\prime \prime}\left(\chi^{\prime}+i \chi^{\prime \prime}\right) .
$$

The atomic part of the anomalous scattering $\Delta f_{a}=f_{a}^{\prime}+i f_{a}^{\prime \prime}$ includes contributions from all shells with excitation energies below the incident photon energy, but does not include the photoelectron scattering fine-structure. The coefficient of the fine structure $f_{r}^{\prime \prime}$ is the resonant part of $f_{a}^{\prime \prime}$ which is proportional to $\mu_{0}$ in XAFS due solely to the excited core electron and is zero below the absorption edge. The total $f_{a}^{\prime \prime}$, by comparison, is proportional to $\mu_{\text {tot }}(E)$ in XAFS and often contains substantial non-zero contributions from electrons in the off-resonance orbitals.

During a DAFS measurement, the direction of the incident and outgoing photons with respect to the unit cell changes as a function of energy according to Bragg's law. In order to use Eq. (4) for site separation, the same polarization dependence must be maintained in the fine structure throughout several DAFS measurements so that the site-specific $\Delta f(E)$ are independent of $\mathrm{Q}$. When the outgoing photon polarization sense is not rotated by the resonant scattering, the fine structure from each site can be kept Q-independent by rotating the crystal about an axis parallel to the incident photon polarization. This has the effect of holding the polarization vector in the same orientation with respect to the crystal throughout the scan. However, in the presence of scattering events that rotate the sense of polarization, the projection of the outgoing rotated photon polarization $\hat{e}^{\prime}$ onto the neighboring bonds can give Q-dependence to some features of the spectrum.

A second condition for applying Eq. (4) is that the matrix $W$ of coefficients $W_{j}(\mathbf{Q})$ has an inverse. This is equivalent to the requirement that the Fourier components of the electron density of the resonant atoms projected onto the axis defined by $\hat{\mathbf{Q}}$ are separable. As a specific case, consider the (00 $)$ ) reflections from $\mathrm{YBa}_{2} \mathrm{Cu}_{3} \mathrm{O}_{7} . \mathrm{Along}$ the $\hat{\mathbf{c}}$ axis, the projected densities of the three $\mathrm{Cu}$ atoms are well separated and therefore DAFS data with $\mathbf{Q}$ in any plane that contains $\hat{\mathbf{c}}$ can be used to produce the independent $\mathrm{Cu}(1)$ and $\mathrm{Cu}(2)$ anomalous scattering amplitudes. The resulting functions $f^{\prime \prime}(E)$ are equivalent to $a$ - or $b$-polarized XAFS. On the other hand, for DAFS measured with $\mathbf{Q}$ perpendicular to $\hat{\mathbf{c}}$, the projected densities of the $\mathrm{Cu}$ atoms lie exactly on top of each other and cannot be distinguished.

\section{CONCLUSIONS}

The polarization dependence of resonant diffraction is a very important consideration for all DAFS experiments and for interpreting DAFS data. The full polarization dependence is described by a polarization tensor, however, when the incident beam is linearly polarized and measurements are confined to diffraction peaks in the plane perpendicular to the incident polarization direction, the fine structure can be approximated by a scalar in energy. The tensor nature of resonant $x$-ray diffraction should always be taken into account for crystallographic refinement of $\triangle f(E)$ from DAFS data, with particular attention to the fine-structure when the measurements are made using image plates or by powder diffraction integrated over the powder ring, since both of these geometries include out-of-plane diffraction.

\section{References}

[1] Zabinsky S. I., Rehr J. J., Ankudinov A., Albers R. C., Eller M. J., Phys. Rev. B 52 (1995) 2995-3009.

[2] Pickering I. J., Sansone M., Marsch J., and George G. N., J. Am. Chem. Soc. 115 (1993) 6302-6311. 